\title{
Widespread Modulation of Cerebral Perfusion Induced during and after Transcranial Direct Current Stimulation Applied to the Left Dorsolateral Prefrontal Cortex
}

\author{
Charlotte J. Stagg, ${ }^{1 \star}$ Richard L. Lin, ${ }^{1,2 \star}$ Melvin Mezue, ${ }^{1,2}$ Andrew Segerdahl, ${ }^{1,2}$ Yazhuo Kong, ${ }^{1}$ Jingyi Xie, ${ }^{1}$ \\ and Irene Tracey ${ }^{1,2}$ \\ ${ }^{1}$ Oxford Centre for Functional Magnetic Resonance Imaging of the Brain and ${ }^{2}$ Nuffield Division of Anaesthetics, Nuffield Department of Clinical \\ Neurosciences, University of Oxford, John Radcliffe Hospital, Oxford OX3 9DU, United Kingdom
}

\begin{abstract}
Noninvasive neuromodulatory techniques such as transcranial direct current stimulation (tDCS) are attracting increasing interest as potential therapies for a wide range of neurological and psychiatric conditions. When targeted to the dorsolateral prefrontal cortex (DLPFC), anodal, facilitatory tDCS has been shown to improve symptoms in a range of domains including working memory, mood, and pain perception (Boggio et al., 2008a; Dockery et al., 2009; Kalu et al., 2012). However, the mechanisms underlying these promising behavioral effects are not well understood. Here, we investigated brain perfusion changes, as assessed using whole-brain arterial spin labeling (ASL), during tDCS applied to the left DLPFC in healthy humans. We demonstrated increased perfusion in regions closely anatomically connected to the DLPFC during anodal tDCS in conjunction with a decreased functional coupling between the left DLPFC and the thalami bilaterally. Despite highly similar effects on cortical excitability during and after stimulation (Nitsche and Paulus, 2000, 2001), cortical perfusion changes were markedly different during these two time periods, with widespread decreases in cortical perfusion being demonstrated after both anodal and cathodal tDCS compared to the period during stimulation. These findings may at least partially explain the different effects on behavior in these time periods described previously in the motor system (Stagg et al., 2011). In addition, the data presented here provide mechanistic explanations for the behavioral effects of anodal tDCS applied to the left DLPFC in terms of modulating functional connectivity between the DLPFC and thalami, as has been hypothesized previously (Lorenz et al., 2003).
\end{abstract}

\section{Introduction}

Noninvasive neuromodulatory techniques are increasingly being studied as potential adjunct therapies for a wide range of neurological and psychiatric conditions including pain, depression, and stroke rehabilitation (Hummel et al., 2005; Fregni et al., 2006b; Boggio et al., 2008b). Of these, transcranial direct current stimulation (tDCS), shows special promise as it is well tolerated and capable of inducing behavioral aftereffects lasting for at least 3 months (Reis et al., 2009). tDCS involves passing a constant 1-2 $\mathrm{mA}$ electric current for 10-20 min via two large electrodes placed on the scalp, it can be targeted to a specific brain region via electrode placement (Nitsche and Paulus, 2000), and the effects of tDCS are polarity specific (Nitsche and Paulus, 2000).

\footnotetext{
Received Aug. 14, 2012; revised April 10, 2013; accepted April 13, 2013

Author contributions: C.J.S., R.L.L., and I.T. designed research; C.J.S., R.L.L., M.M., and J.X. performed research; C.J.S., R.L.L., A.S., Y.K., and J.X. analyzed data; C.J.S., R.L.L., and I.T. interpreted the data and wrote the paper.

This work was supported by the Marshall Aid Commemoration Commission (R.L.L.) and the Wellcome Trust and Medical Research Council (A.S., I.T.). I.T. and C.J.S. are grateful to the National Institute for Health Research (NIHR) Oxford Biomedical Research Centre based at Oxford University Hospitals Trust, Oxford University, for support. The views expressed are those of the author(s) and not necessarily those of the NHS, the NIHR, or the Department of Health.

The authors declare no competing financial interests.

*C.J.S. and R.L.L. contributed equally to this work.

Correspondence should be addressed to Dr. Charlotte Stagg, Oxford Centre for Functional Magnetic Resonance Imaging of the Brain, John Raddliffe Hospital, Headington, Oxford OX3 9DU, UK. E-mail: cstagg@fmrib.ox.ac.uk.

DOI:10.1523/JNEUROSCI.3887-12.2013

Copyright $\odot 2013$ the authors $\quad 0270-6474 / 13 / 3311425-07 \$ 15.00 / 0$
}

tDCS to the dorsolateral prefrontal cortex (DLPFC) has behavioral consequences on working memory, decision making, depression, and pain (Fregni et al., 2006a; Fecteau et al., 2007; Boggio et al., 2008a; Dockery et al., 2009; Kalu et al., 2012), making it an important potential therapeutic tool in a variety of conditions.

Previous studies have investigated the anatomical distribution of primary motor cortex (M1) tDCS, with the second electrode over the contralateral supraorbital ridge, using fMRI or PET to image the effects of tDCS on task performance (Baudewig et al., 2001; Lang et al., 2005; Stagg et al., 2009) or on the resting brain (Lang et al., 2005; Polanía et al., 2011; Zheng et al., 2011). In the main, these studies suggest that tDCS modulates cortical activity both within the region directly under the electrode and in closely anatomically connected but spatially distant regions. However, it is difficult to directly extrapolate these findings to therapeutic DLPFC stimulation as modeling studies suggest the effects of tDCS are highly dependent on underlying sulcal anatomy (Datta et al., 2009).

Additionally, although the cortical excitability changes induced by tDCS are virtually indistinguishable during stimulation and in the poststimulation period (Nitsche and Paulus, 2000, 2001), the intracortical underpinnings of these effects differ considerably (Nitsche et al., 2005), with the effects of tDCS during stimulation largely driven by direct effects on membrane polarity while changes after stimulation involve modulation of GABAer- 
gic and glutamatergic synapses (see Stagg and Nitsche (2011)). However, no systematic investigation comparing cortical blood flow changes between these two time windows has yet been performed.

Here, we investigate cortical perfusion during and after 20 min of left DLPFC (L-DLPFC) tDCS using the novel fMRI approach of arterial spin labeling (ASL). ASL is primarily sensitive to low-frequency signals (Aguirre et al., 2002) and is therefore the ideal modality to detect blood flow changes induced by the minutes-long tDCS protocols commonly used. We wished to study both the spatial and temporal characteristics of L-DLPFC tDCS to inform future studies of tDCS. It is important to note that though we refer here to L-DLPFC tDCS, the effects of this tDCS are likely driven by both the electrodes, and therefore should be considered to be effects of the montage as a whole.

\section{Materials and Methods}

Twenty-four healthy right-handed subjects gave their informed consent to participate in the study [Experiment 1, 12 subjects; 4 female; age (mean \pm SD), $25.7 \pm 4.7$ years; Experiment 2, 12 subjects; 2 female; age, $27.7 \pm 5.7$ years $]$. Subjects were excluded if they had any neurological or psychiatric disorders or contraindications to MRI or tDCS (Nitsche et al., 2008).

\section{Experiment 1}

All subjects participated in two experimental sessions, one with anodal tDCS and one with cathodal tDCS to L-DLPFC. The session order was counterbalanced across the group, and sessions were separated by at least 1 week. Subjects were blinded as to the stimulation direction used.

tDCS was applied during fMRI acquisition using an MR-compatible system (DC-Stimulator MR, Magstim). As in previous tDCS studies of the L-DLPFC, one electrode was centered on F3 (Fregni et al., 2006a; Boggio et al., 2008a; Boggio et al., 2008b), with the second electrode on the contralateral supraorbital ridge. Two $5 \times 7 \mathrm{~cm}$ electrodes with $5 \mathrm{k} \Omega$ resisters were used with high-chloride EEG gel (Abralyt $\mathrm{HiCl}$, EasyCap). Twenty minutes of $1 \mathrm{~mA}$ tDCS was given with fade-in/fade-out periods of $10 \mathrm{~s}$.

\section{Experiment 2}

All subjects participated in one experimental session with sham tDCS to L-DLPFC. Sham tDCS was applied as in Experiment 1, but was switched off after $30 \mathrm{~s}$.

\section{Image acquisition}

Pseudocontinuous ASL was acquired on a 3T MRI system (Magnetom Verio 3T, Siemens) using a 32-channel head coil. The ASL sequence used GRAPPA (with a factor of 2) and applied a gradient-echo EPI readout (TR, $3.21 \mathrm{~s}$; TE, $11 \mathrm{~ms}$; 7/8 $k$ space). Twenty-eight axial slices were acquired in ascending order $(4 \times 4 \times 4.6 \mathrm{~mm}$ voxels $)$ with an interslice gap of $0.46 \mathrm{~mm}$ to give whole brain coverage. Using a time-of-flight scan of the neck, we chose the optimal labeling plane for each subject at $\sim 8$ to 10 $\mathrm{cm}$ inferior to the center of the axial slices. Each labeling pulse lasted $1.4 \mathrm{~s}$ and was followed by a postlabeling delay of $900 \mathrm{~ms}$. A high-resolution T1-weighted structural image was acquired (MPRAGE; TR, $2040 \mathrm{~ms}$; TE, $4.68 \mathrm{~ms}$; 192 axial slices; $1 \times 1 \times 1 \mathrm{~mm}$ resolution).

In each experimental session, a 10 min baseline scan ("baseline") was acquired. We then performed concurrent tDCS-fMRI for $20 \mathrm{~min}$ ("stimulation"), followed by a 10 min acquisition after tDCS ("aftereffects").

\section{Image analysis}

Analysis was performed using tools from the FMRIB software library version 4.1.7 (http://www.fmrib.ox.ac.uk/fsl) (Smith et al., 2004; Woolrich et al., 2009). Standard preprocessing steps were applied: motion correction using MCFLIRT (Jenkinson et al., 2002), nonbrain tissue removal using Brain Extraction Tool (BET) (Smith, 2002), nonlinear highpass temporal filtering ( $\sigma=200$ s) (Chuang et al., 2008), and spatial smoothing with a Gaussian kernel of $8 \mathrm{~mm}$ full-width at half-maximum (Zheng et al., 2011). Time-series statistical analysis was performed using FMRIB's Improved Linear Model (FILM) with local autocorrelation cor- rection (Woolrich et al., 2001). Functional data were registered to each subjects' high-resolution T1 structural scan and then to the Montreal Neurological Institute standard brain using FMRIB's linear registration tool (FLIRT) (Jenkinson et al., 2002).

At the first level, individual subject's perfusion time series were generated by fitting a model of the relevant "tag" and "control" images to the preprocessed four-dimensional data sets. Time series data within each voxel were fit to the perfusion model to yield a resultant parameter estimate image that was proportional to localized blood flow. To determine the percentage signal change in cerebral blood flow $(\mathrm{CBF})$ relative to each experimental condition within each subject, a fixed-effects second-level analysis was performed for each subject separately, whereby a model of the baseline, stimulation, and aftereffects for both the anodal and cathodal tDCS sessions was convolved to each subject's perfusion time series. At the third level, a mixed-effects analysis was used to obtain group mean activation maps for the main effect of each stimulus condition on changes in CBF across the whole brain. Cluster-based thresholding was used to find clusters of $\mathrm{CBF}$ changes showing effects with a significance level of $Z>2.0$ and $p<0.01$. This sequence of analyses allowed us to take into account both the differences in variances between the three scans performed on the same day as well as differences across scan sessions performed on different days (Stagg et al., 2009).

\section{Functional connectivity analysis}

To investigate changes in coupling from the L-DLPFC to other brain regions, a functional connectivity analysis was performed. We first defined masks for the L-DLPFC and the right frontopolar cortex (R-FPC), the cortical region under the electrode placed over the right supraorbital ridge.

$L$-DLPFC. The L-DLPFC was based on cytoarchitectonic features that included Brodmann areas 8A, 46, and 9/46, which have a well-developed layer IV (Petrides and Pandya, 1999; Petrides, 2005). Area 9/46 was located on the middle frontal gyrus and the inferior portion of the superior frontal gyrus. Area 46 consisted of the middle part of the middle frontal gyrus, anteriorly adjacent to area 10 (Petrides and Pandya, 1999). The closely related Brodmann areas 9 and $8 \mathrm{~B}$ were excluded due to the sparse granular layer in their cytoarchitecture.

$R$-FPC. The R-FPC was based on cytoarchitectonic features that included the lateral section of Brodmann area 10, consisting of the anterior portions of the middle and superior frontal gyri (Christoff and Gabrieli, 2000). The superior and inferior borders are demarcated by the dorsolateral prefrontal and the orbitofrontal cortices, respectively.

For the whole-brain analysis, standard preprocessing steps were applied to the fMRI data as detailed above. Next, the perfusion time course was derived using in-house scripts on a voxel-by-voxel basis, and the mean perfusion time course within the L-DLPFC and R-FPC masks was extracted for each mask and each scan separately and used as first-level regressors for each subject and time period separately. Group-level analyses were subsequently performed as described above.

\section{Results}

\section{Brain perfusion changes during stimulation}

To characterize the brain perfusion changes induced by tDCS, we first investigated the differences in whole-brain perfusion between the baseline and the stimulation scans [i.e., $\mathrm{tDCS}_{\text {stimulation }}-$ $\left.\left.\mathrm{tDCS}_{\text {baseline }}\right)\right]$. Anodal $\mathrm{tDCS}$ led to an increase in perfusion in the left primary sensory cortex (S1), midcingulate cortex, paracingulate cortex, and left parietal cortex compared with baseline (Fig. 1A). Cathodal $\mathrm{tDCS}$ led to a decrease in perfusion in the thalami bilaterally, and the right middle and inferior temporal gyri compared with baseline (Fig. 1B).

To confirm that these changes were not driven by nonspecific temporal drifts, we performed identical analyses on the sham experiment. No significant differences were seen between the sham stimulation and baseline or aftereffects time periods. To ensure that these differences were not driven by differences in the baseline between the two stimulation conditions, we directly compared the baseline sessions (i.e., anodal $\mathrm{baseline}_{-}-$cathodal $\left._{\text {baseline }}\right)$. No significant differences were demonstrated. 
A
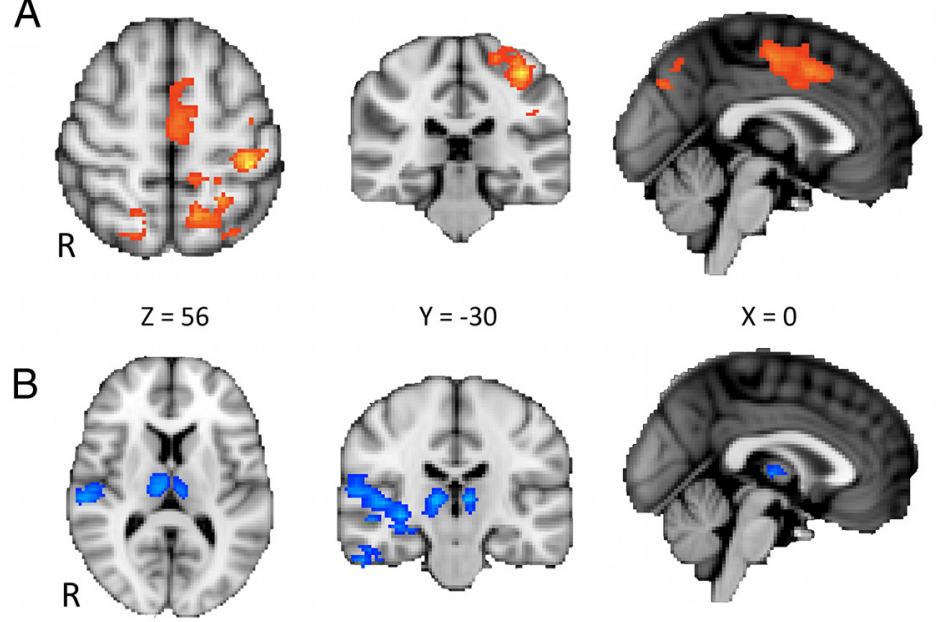

$Z=10$

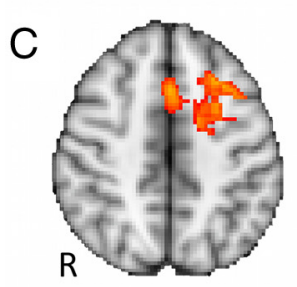

$Z=46$

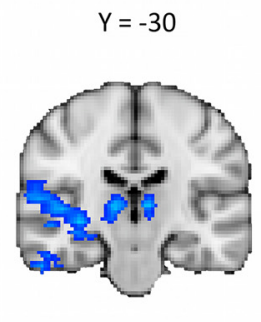

$Y=-22$

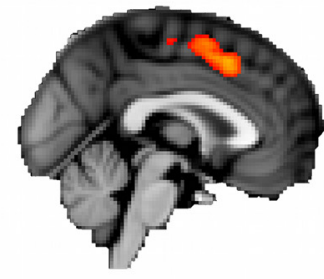

$X=0$
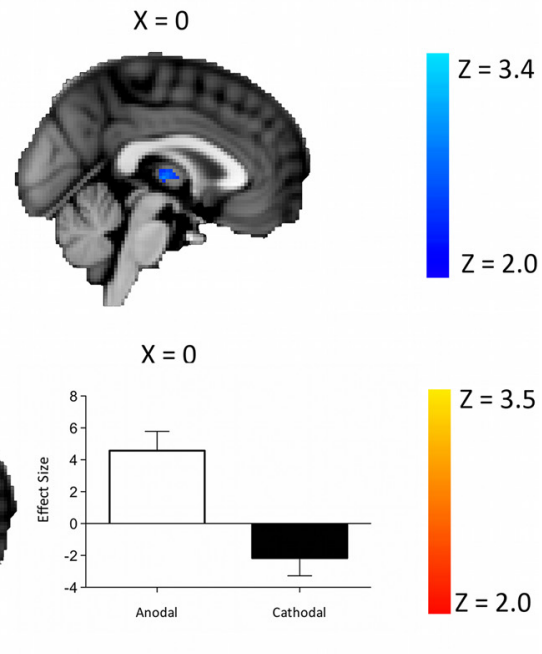

Figure 1. Brain perfusion changes during stimulation compared with baseline ( $n=12$; mixed effects, corrected cluster threshold, $Z>$ $2.0, p<0.01)$. A, Regions of increased perfusion during anodal stimulation [i.e., (anodal ${ }_{\text {stimulation }}-$ anodal $_{\text {baseline }}$ ]. Increases in perfusion were seen in the left primary sensory cortex, midcingulate cortex, paracingulate cortex, and left parietal cortex. There were no regions of

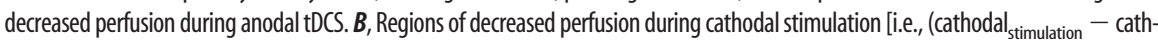
odal $\left._{\text {baseline }}\right)$ ]. Decreases were seen in the thalami bilaterally and the right middle and inferior temporal gyri. No regions of increased perfusion were seen during cathodal tDCS. C, Regions of increased perfusion during anodal tDCS compared with during cathodal tDCS [i.e., $\left(\right.$ anodal $_{\text {stimulation }}$ - anodal $\left.\left.\right|_{\text {baseline }}\right)$ - (cathodal stimulation $_{\text {- cathodal }}$ baseline) $)$. Increased perfusion was seen in the L-DLPFC and the paracingulate cortex. The perfusion changes within these regions are shown on the right. No regions of increased perfusion during cathodal tDCS compared with during anodal tDCS were seen. Error bars indicate SEM. R, Right.

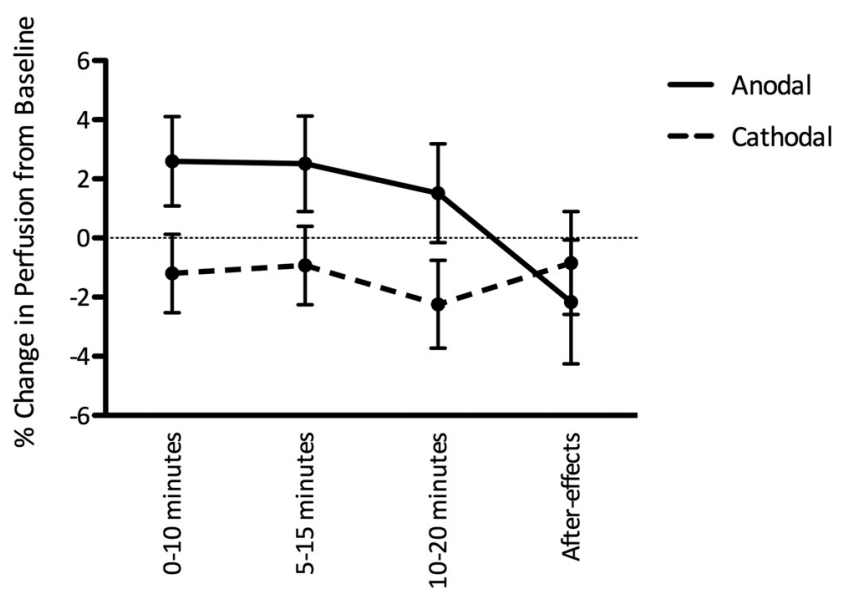

Figure 2. Time course of perfusion changes during and after stimulation within the L-DLPFC. A sustained increase in perfusion during anodal tDCS and a decrease in perfusion during cathodal tDCS were observed. Error bars indicate SEM.

To investigate polarity specific effects on whole-brain cerebral perfusion, we directly contrasted the effects of anodal and cathodal stimulation [i.e., (anodal $l_{\text {stimulation }}-$ anodal $_{\text {baseline }}$ ) compared to (cathodal $l_{\text {stimulation }}-$ cathodal $\left._{\text {baseline }}\right)$ ]. Increased perfusion with anodal stimulation compared with cathodal tDCS

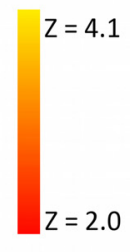

was demonstrated in the left, stimulated DLPFC and the paracingulate cortex (Fig. $1 C)$. No regions of increased perfusion were demonstrated with cathodal tDCS compared with anodal tDCS.

To directly investigate the cortical perfusion changes under the stimulating electrode, we performed an ROI-based analysis of the L-DLPFC. Comparing the change in perfusion from baseline in both the stimulation and aftereffect periods for anodal and cathodal tDCS, we saw a trend toward a polarity-specific effect of tDCS (main effect of polarity, $F_{(1,11)}=4.05, p=$ 0.06 ), which varied between the stimulation and aftereffects scans (time by polarity interaction, $\left.F_{(1,11)}=6.80, p=0.024\right)$.

To investigate the temporal evolution of the perfusion changes during stimulation, we then divided the stimulation data within the L-DLPFC ROI into three 10 min blocks $(0-10,5-15$, and $10-20 \mathrm{~min}$; Fig. 2). During tDCS, there was a trend toward a polarity-specific increase in perfusion with anodal tDCS and decreased with cathodal tDCS (main effect of polarity, $\left.F_{(1,11)}=3.64, p=0.08\right)$, but this did not vary significantly with time (main effect of time, $F_{(2,22)}=1.60, p=0.22$; time by polarity interaction, $F_{(2,22)}=0.03, p=$ $0.96)$, suggesting that the perfusion changes were stable across the $20 \mathrm{~min}$ of stimulation for both anodal and cathodal tDCS.

We went on to investigate changes in functional connectivity between the stimulated DLPFC and other regions. During anodal tDCS, there was an increase in coupling between the L-DLPFC and the R-DLPFC and the left sensorimotor cortex, and a decrease in coupling between the L-DLPFC and the thalami bilaterally, the brain stem, and the cerebellum (Fig. 3A). During cathodal tDCS there was a decrease in coupling between the L-DLPFC and an extensive region of the left temporal, parietal, and occipital lobes (Fig. 3B). No changes in functional connectivity were observed between the R-FPC and any other brain regions during either anodal or cathodal tDCS.

\section{Brain perfusion changes after stimulation}

Neurophysiologically, the magnitudes of the cortical excitability changes induced by tDCS, as measured via transcranial magnetic stimulation-evoked potentials, are highly similar during stimulation and in the immediate afterstimulation period (Nitsche et al., 2005). To explore the brain perfusion changes associated with these excitability shifts, we investigated the differences in wholebrain perfusion between the stimulation and the aftereffects periods [i.e., $\left.\left(\mathrm{tDCS}_{\text {aftereffects }}-\mathrm{tDCS}_{\text {stimulation }}\right)\right]$. There was a widespread decrease in perfusion in the frontal lobes bilaterally, the cerebellum, and the precuneus after anodal tDCS compared with during stimulation (Fig. 4A). To explore whether cortical perfusion differed within these regions between the baseline and stimulation scans (a question orthogonal to our initial investigation of the data), we performed a post hoc $t$ test that showed no significant differences within these regions be- 
tween baseline and stimulation (paired $t$ test, $\left.t_{(11)}=0.85, p=0.41\right)$.

A widespread decrease in perfusion in the occipital cortices and the cerebellum was demonstrated after cathodal tDCS compared with during stimulation (Fig. $4 B$ ). Again there was no change in perfusion in these regions between the baseline and stimulation periods $\left(t_{(11)}=0.51, p=\right.$ $0.61)$. No regions of increased perfusion after either anodal tDCS or cathodal tDCS were observed.

It is not clear how a polarity-specific contrast could be interpreted in this case. To investigate non-polarity-specific perfusion changes after anodal and cathodal stimulation compared with during tDCS, we performed a conjunction analysis [i.e., (anodal ${ }_{\text {aftereffects }}-$ anodal $_{\text {stimulation }}$ ) in addition to (cathodal aftereffects - $_{\text {- }}$ cathodal stimulation $)$. After both anodal and cathodal tDCS stimulation, there was a decrease in perfusion within the cuneus, lingual gyrus, and primary visual cortices compared with during stimulation (Fig. 4C).

We then compared perfusion changes after stimulation with those during the baseline condition [i.e., $\left(\mathrm{tDCS}_{\text {aftereffects }}-\right.$ $\left.\left.\mathrm{tDCS}_{\text {baseline }}\right)\right]$. There were no regions of significantly increased activity after anodal stimulation compared with baseline. Significant decreases in perfusion were seen after anodal stimulation compared with baseline in the frontal lobes and the precuneus bilaterally (Fig. 5A). There were no regions of significantly increased activity after cathodal stimulation compared with baseline. Significant decreases in perfusion were seen after cathodal stimulation compared with baseline in the frontal and temporal lobes bilaterally (Fig. 5B).

A previous study demonstrated increased coupling between the stimulated DPLFC and connected regions after anodal tDCS using BOLD fMRI (Keeser et al., 2011). To compare our results to this previous study, we contrasted functional connectivity after stimulation to baseline [i.e., $\left(\mathrm{tDCS}_{\text {aftereffects }}-\mathrm{tDCS}_{\text {baseline }}\right)$ ]. Increased coupling with the L-DLPFC was demonstrated after anodal tDCS compared with baseline in the primary sensorimotor cortices bilaterally (Fig. 6). No changes in coupling with the L-DLPFC were demonstrated after cathodal tDCS.

\section{Discussion}

This study was designed to answer two specific questions as to the optimal use of L-DLPFC $t D C S$ as a therapeutic intervention: What effect does tDCS applied to the
A

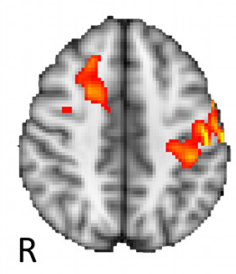

$Z=44$

\section{B}

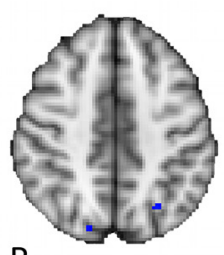

$Z=44$

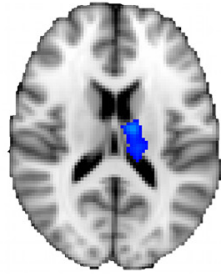

$Z=16$

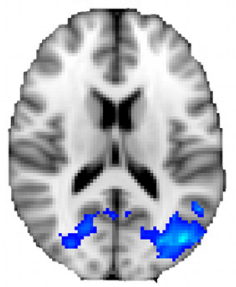

$Z=16$
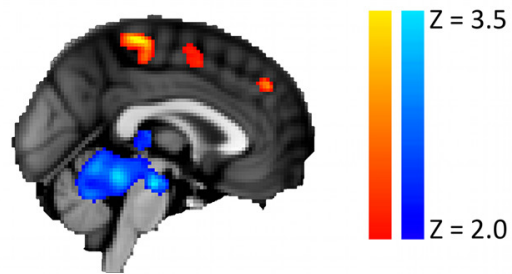

$$
x=0
$$
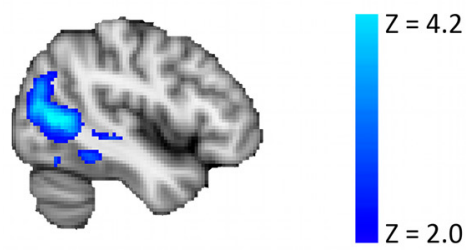

$X=-44$
Figure 3. Functional connectivity analysis from the stimulated L-DLPFC ( $n=12$; mixed effects, corrected cluster threshold, $Z>2.0, p<0.01$ ). A, Regions of altered functional connectivity during anodal stimulation [i.e., (anodal stimulation $_{\text {- }}$ anodal $\left._{\text {baseline }}\right)$ ]. Significant increases in connectivity (in red/yellow) were seen between the L-DLPFC and the R-DLPFC and the left sensorimotor cortex. A significant decrease in connectivity (in blue) was seen between the L-DLPFC and the thalami bilaterally. B, Regions of decreased functional connectivity during cathodal stimulation [i.e., (cathodal stimulation $_{\text {- cath- }}$ odal $\left.\left.{ }_{\text {baseline }}\right)\right]$. Decreased functional connectivity was observed between the L-DLPFC and an extensive region in the left temporal, parietal, and occipital lobes. R, Right.
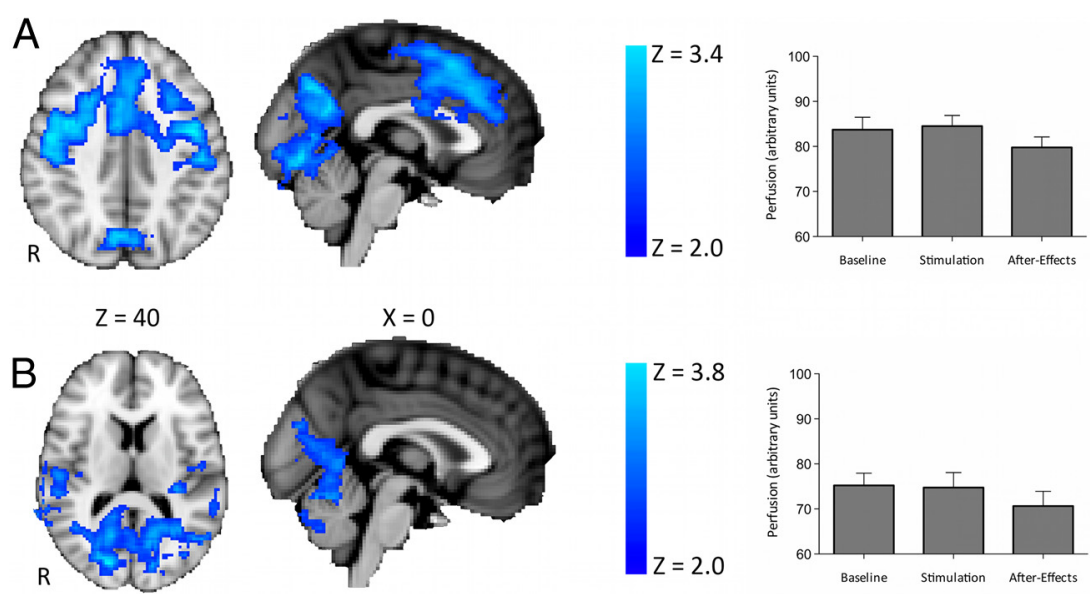
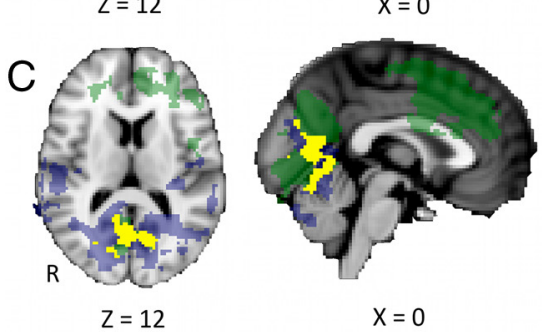

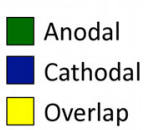

Figure 4. Regions of decreased perfusion after stimulation compared with during stimulation [i.e., $\left(\mathrm{tDCS}_{\text {aftereffects }}-\right.$ $\mathrm{tD}\left(S_{\text {stimulation }}\right) ; n=12$; mixed effects, corrected cluster threshold, $\left.Z>2.0, p<0.01\right]$. $A$, Regions of decreased perfusion after anodal tDCS. Decreased perfusion can be seen in the frontal lobes bilaterally, the cerebellum, and the precuneus. Mean cortical perfusion within these regions at each time point is shown on the right. $\boldsymbol{B}$, Regions of decreased perfusion after cathodal tDCS. Decreased perfusion can be seen in widespread regions, including the occipital cortices and the cerebellum. Mean cortical perfusion within these regions at each time point is shown on the right. C, Conjunction analyses of $\boldsymbol{A}$ and $\boldsymbol{B}$ [i.e., (anodal aftereffects - anodal $\left._{\text {stimulation }}\right)+\left(\right.$ cathodal $_{\text {aftereffects }}-$ cathodal $\left._{\text {stimulation }}\right)$ ]. Both anodal and cathodal tDCS led to a decrease in perfusion within the cuneus, lingual gyrus, and primary visual cortices compared with during stimulation. R, Right. 
A
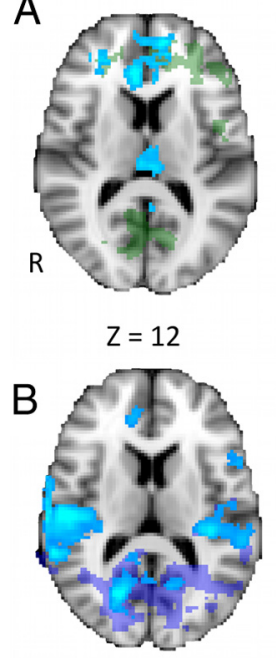

$Z=12$
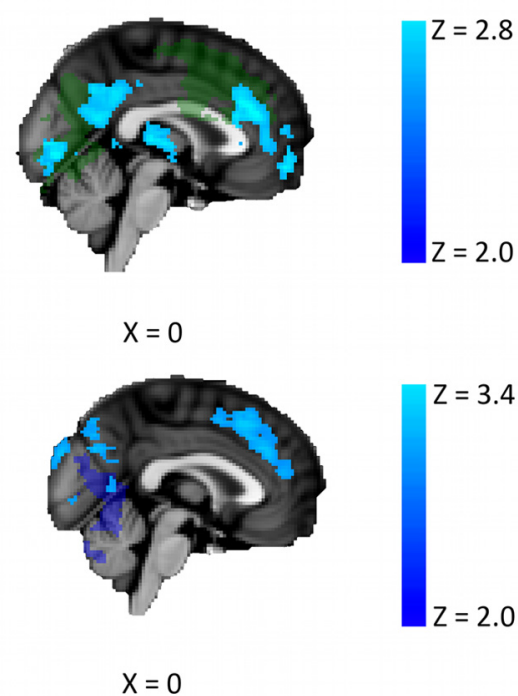

Figure 5. Regions of decreased perfusion after stimulation compared with during stimulation [i.e., ( $\mathrm{tDCS}$ aftereffects $-\mathrm{tDCS}$ baseline); $n=12$; mixed effects, corrected cluster threshold, $Z>$ $2.0, p<0.01] . A$, Regions of decreased perfusion after anodal tDCS. Decreased perfusion can be seen in the frontal lobes and the precuneus bilaterally. For ease of comparison, the results of the contrast (anodal $\mathrm{aftereffects}-$ anodal $_{\text {stimulation }}$ ) are shown in green. $\boldsymbol{B}$, Regions of decreased perfusion after cathodal tDCS. Decreased perfusion can be seen in the frontal and temporal lobes bilaterally. For ease of comparison, the results of the contrast (cathodal $_{\text {aftereffects }}$ - cathodal $_{\text {stimulation }}$ ) are shown in purple. R, Right.

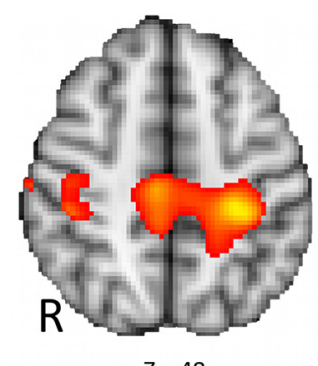

$Z=48$

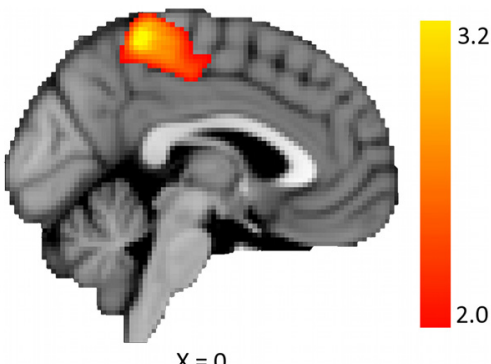

$X=0$
Figure 6. Functional connectivity analysis from the stimulated L-DLPFC $(n=12$; mixed effects, corrected cluster threshold, $Z>2.0, p<0.01$ ), showing regions where coupling is altered after stimulation compared with before [i.e., (anodal ${ }_{\text {aftereffects }}-$ anodal $_{\text {baseline }}$ )]. Increased coupling after anodal tDCS compared with baseline was observed in the primary sensorimotor cortices bilaterally. R, Right.

DLPFC have on cortical perfusion patterns? And do the cortical perfusion changes seen during stimulation continue after stimulation has ceased?

We demonstrated widespread perfusion changes associated with both anodal and cathodal tDCS applied to L-DLPFC. During stimulation, anodal tDCS, which increases cortical excitability, led to increased perfusion within brain regions closely structurally connected to the L-DLPFC. Cathodal tDCS, which decreases cortical excitability, led to decreased perfusion within the thalami bilaterally. The effects of stimulation on the stimulated, left DLPFC were polarity specific: directly contrasting perfusion changes with anodal and cathodal tDCS demonstrated polarity-specific perfusion increases during anodal tDCS compared with cathodal stimulation within the stimulated region. This finding is in line with animal studies suggesting an increase in perfusion with anodal tDCS and a decrease with cathodal tDCS under the stimulating electrode (Wachter et al., 2011).
The DLPFC has been suggested previously as a therapeutic target in chronic pain because activity here correlates negatively with the perceived intensity and unpleasantness of a painful stimulus, an effect hypothesized to be mediated by a top-down inhibition of the midbrain-thalamic pathway (Lorenz et al., 2003). Interventions such as anodal tDCS, which increase activity within the DLPFC, have been demonstrated to alleviate painful experiences (Boggio et al., 2008a; Short et al., 2011), putatively via an indirect, inhibitory effect on the thalamus, which in turn likely influences descending pain modulatory systems in the brainstem. The decreased coupling between L-DLPFC and thalamus observed here during anodal tDCS supports this role for the DLPFC and offers a possible mechanistic explanation for at least some of the analgesic effects of anodal stimulation. However, as with all tDCS experiments, the effects of L-DLPFC tDCS demonstrated here are likely to be specific to the tDCS montage used. We chose this tDCS montage because it has most commonly been used for previous studies, but it may be that placing the right orbitofrontal electrode in another position would result in different effects.

We used ASL to investigate the effects of tDCS on the brain as it is primarily sensitive to low-frequency signals and can therefore be used to investigate perfusion changes both during and after a therapeutically relevant stimulation paradigm. One previous study used ASL to investigate perfusion changes during tDCS targeted to M1 (Zheng et al., 2011). Zheng et al. (2011) demonstrated a trend toward an increase in perfusion during anodal tDCS within the stimulated cortex, as seen here, their results only becoming significant when compared to changes induced within a control brain region. They also observed an increase in perfusion during cathodal tDCS, albeit smaller than that after anodal tDCS. However, it is difficult to directly compare the results of this previous study with those presented here as Zheng et al. (2011) studied the effects of repeated, short sessions of tDCS. Cortical excitability changes induced by repeated tDCS interventions are more complex than those induced by a single stimulation period (Fricke et al., 2011), and a cathodal tDCS protocol similar to that used by Zheng et al. (2011) was demonstrated previously to lead to an increase in cortical excitability (Fricke et al., 2011), making interpretation of these results complex.

A previous BOLD fMRI study of anodal DLPFC tDCS demonstrated an increase in resting functional connectivity after stimulation within networks that included the DLPFC compared with baseline (Keeser et al., 2011). Here, we saw increases in functional connectivity due to anodal tDCS between the L-DLPFC and the primary sensorimotor cortices bilaterally. This discrepancy may be explained by the differing acquisition and analysis approaches of these two studies, but the finding that tDCS leads to an increase coupling between L-DLPFC and M1 may add a mechanistic explanation for the analgesic effects of anodal M1 tDCS (Fregni et al., 2006a,b; Boggio et al., 2008a).

The polarity-specific cortical excitability changes induced by tDCS are highly similar during stimulation and in the immediate afterstimulation period (Nitsche et al., 2005) at least within M1, when a single application of up to $2 \mathrm{~mA}$ and $20 \mathrm{~min}$ is adhered to. All previous L-DLPFC tDCS studies referenced here used stimulation parameters within these limits, but longer durations of anodal stimulation have been demonstrated to lead to a decrease in cortical excitability (Monte-Silva et al., 2013), suggesting that the effects of tDCS may be critically dependent on parameters of stimulation used.

Although not studied in depth for the electrode montage used here, the previously described behavioral effects of tDCS in the classical M1 montage (Nitsche and Paulus, 2000) during these 
time periods are very different. During stimulation, anodal tDCS leads to an improvement in a number of different motor learning paradigms (Nitsche et al., 2003; Reis et al., 2009; Galea and Celnik, 2009; Stagg et al., 2011). After stimulation, anodal tDCS leads either to a decrement in learning (Stagg et al., 2011) or has no effect (Kuo et al., 2008). Cathodal tDCS has variously been shown to worsen motor learning, both during and after stimulation (Stagg et al., 2011), or to have no significant effects (Nitsche et al., 2003; Reis et al., 2009).

In line with these differing behavioral effects, the cortical perfusion changes induced by tDCS during and after stimulation were significantly different. We demonstrated widespread decreases in cortical perfusion after anodal tDCS compared with during stimulation in an anatomical distribution similar to that of the default mode network (DMN). Direct post hoc testing revealed no significant increase in perfusion within these regions during stimulation compared with baseline. This decrease in perfusion within regions associated with the DMN after anodal tDCS might suggest that an increase in cortical excitability within the DLPFC induced by anodal tDCS leads to a subsequent disturbance of the integrity of the DMN. Whatever the underlying mechanism, importantly for the purposes of using anodal tDCS as a potential adjunctive therapy, this finding would suggest that the timing of $\mathrm{tDCS}$ with another intervention may be important when considering the potential therapeutic benefits of tDCS, although the exact timing dependence has yet to be fully explored.

In conclusion, to our knowledge, this study is the first to examine cortical perfusion changes associated with L-DLPFC tDCS, a potential therapeutic target for conditions including chronic pain. We have demonstrated widespread cortical perfusion and functional coupling changes in regions closely anatomically connected to the DLPFC during anodal tDCS, in particular a decrease in functional connectivity with the bilateral thalami, adding weight to the hypothesis that the DLPFC modulates pain via a decrease in thalamic activity. The results presented in this paper add to a conceptual framework on the mechanisms of tDCS and inform our use of tDCS targeted to the DLPFC as a potential adjunctive therapy.

\section{References}

Aguirre GK, Detre JA, Zarahn E, Alsop DC (2002) Experimental design and the relative sensitivity of BOLD and perfusion fMRI. Neuroimage 15: 488-500. CrossRef Medline

Baudewig J, Nitsche M, Paulus W, Frahm J (2001) Regional modulation of BOLD MRI responses to human sensorimotor activation by transcranial direct current stimulation. Mag Res Med 45:196-201. CrossRef

Boggio PS, Zaghi S, Lopes M, Fregni F (2008a) Modulatory effects of anodal transcranial direct current stimulation on perception and pain thresholds in healthy volunteers. Eur J Neurol 15:1124-1130. CrossRef Medline

Boggio PS, Rigonatti SP, Ribeiro RB, Myczkowski ML, Nitsche MA, PascualLeone A, Fregni F (2008b) A randomized, double-blind clinical trial on the efficacy of cortical direct current stimulation for the treatment of major depression. Int J Neuropsychopharmacol 11:249-254. Medline

Christoff K, Gabrieli J (2000) The frontopolar cortex and human cognition: Evidence for a rostrocaudal hierarchical organization within the human prefrontal cortex. Psychobiology 28:168-186.

Chuang KH, van Gelderen P, Merkle H, Bodurka J, Ikonomidou VN, Koretsky AP, Duyn JH, Talagala SL (2008) Mapping resting-state functional connectivity using perfusion MRI. Neuroimage 40:1595-1605. CrossRef Medline

Datta A, Bansal V, Diaz J, Patel J, Reato D, Bikson M (2009) Gyri -precise head model of transcranial DC stimulation: Improved spatial focality using a ring electrode versus conventional rectangular pad. Brain Stim 2:201-207. CrossRef

Dockery CA, Hueckel-Weng R, Birbaumer N, Plewnia C (2009) Enhancement of planning ability by transcranial direct current stimulation. J Neurosci 29:7271-7277. CrossRef Medline
Durand S, Fromy B, Bouyé P, Saumet JL, Abraham P (2002) Vasodilatation in response to repeated anodal current application in the human skin relies on aspirin-sensitive mechanisms. J Physiol 540:261-269. CrossRef Medline

Fecteau S, Knoch D, Fregni F, Sultani N, Boggio P, Pascual-Leone A (2007) Diminishing risk-taking behavior by modulating activity in the prefrontal cortex: a direct current stimulation study. J Neurosci 27:12500-12505. CrossRef Medline

Fregni F, Gimenes R, Valle AC, Ferreira MJ, Rocha RR, Natalle L, Bravo R, Rigonatti SP, Freedman SD, Nitsche MA, Pascual-Leone A, Boggio PS (2006a) A randomized, sham-controlled, proof of principle study of transcranial direct current stimulation for the treatment of pain in fibromyalgia. Arthritis Rheum 54:3988-3998. CrossRef Medline

Fregni F, Boggio PS, Lima MC, Ferreira MJ, Wagner T, Rigonatti SP, Castro AW, Souza DR, Riberto M, Freedman SD, Nitsche MA, Pascual-Leone A (2006b) A sham-controlled, phase II trial of transcranial direct current stimulation for the treatment of central pain in traumatic spinal cord injury. Pain 122:197-209. CrossRef Medline

Fricke K, Seeber AA, Thirugnanasambandam N, Paulus W, Nitsche MA, Rothwell JC (2011) Time course of the induction of homeostatic plasticity generated by repeated transcranial direct current stimulation of the human motor cortex. J Neurophysiol 105:1141-1149. CrossRef Medline

Galea JM, Celnik P (2009) Brain polarization enhances the formation and retention of motor memories. J Neurophysiol 102:294-301. CrossRef Medline

Hummel F, Celnik P, Giraux P, Floel A, Wu WH, Gerloff C, Cohen LG (2005) Effects of non-invasive cortical stimulation on skilled motor function in chronic stroke. Brain 128:490-499. CrossRef Medline

Jenkinson M, Bannister P, Brady M, Smith S (2002) Improved optimisation for the robust and accurate linear registration and motion correction of brain images. Neuroimage 17:825-841. CrossRef Medline

Kalu U, Sexton C, Loo C, Ebmeier K (2012) Transcranial direct current stimulation in the treatment of major depression: a meta-analysis. Psychol Med 1-10.

Keeser D, Meindl T, Bor J, Palm U, Pogarell O, Mulert C, Brunelin J, Möller HJ, Reiser M, Padberg F (2011) Prefrontal transcranial direct current stimulation changes connectivity of resting-state networks during fMRI. J Neurosci 31:15284-15293. CrossRef Medline

Kuo MF, Unger M, Liebetanz D, Lang N, Tergau F, Paulus W, Nitsche MA (2008) Limited impact of homeostatic plasticity on motor learning in humans. Neuropsychologia 46:2122-2128. CrossRef Medline

Lang N, Siebner HR, Ward NS, Lee L, Nitsche MA, Paulus W, Rothwell JC, Lemon RN, Frackowiak RS (2005) How does transcranial DC stimulation of the primary motor cortex alter regional neuronal activity in the human brain? Eur J Neurosci 22:495-504. CrossRef Medline

Lorenz J, Minoshima S, Casey KL (2003) Keeping pain out of mind: the role of the dorsolateral prefrontal cortex in pain modulation. Brain 126:10791091. CrossRef Medline

Monte-Silva K, Kuo MF, Hessenthaler S, Fresnoza S, Liebetanz D, Paulus W, Nitsche MA (2013) Induction of late LTP-like plasticity in the human motor cortex by repeated non-invasive brain stimulation. Brain Stim 6:424-432. CrossRef Medline

Nitsche MA, Paulus W (2000) Excitability changes induced in the human motor cortex by weak transcranial direct current stimulation. J Physiol 527:633-639. CrossRef Medline

Nitsche MA, Paulus W (2001) Sustained excitability elevations induced by transcranial DC motor cortex stimulation in humans. Neurology 57: 1899-1901. CrossRef Medline

Nitsche MA, Schauenburg A, Lang N, Liebetanz D, Exner C, Paulus W, Tergau F (2003) Facilitation of implicit motor learning by weak transcranial direct current stimulation of the primary motor cortex in the human. J Cogn Neurosci 15:619-626. CrossRef Medline

Nitsche MA, Seeber A, Frommann K, Klein CC, Rochford C, Nitsche MS, Fricke K, Liebetanz D, Lang N, Antal A, Paulus W, Tergau F (2005) Modulating parameters of excitability during and after transcranial direct current stimulation of the human motor cortex. J Physiol 568:291-303. CrossRef Medline

Nitsche MA, Cohen LG, Wassermann EM, Priori A, Lang N, Antal A, Paulus W, Hummel F, Boggio PS, Fregni F, Pascual-Leone A (2008) Transcranial direct current stimulation: State of the art 2008. Brain Stim 1:206223. CrossRef

Petrides M (2005) Lateral prefrontal cortex: architectonic and functional 
organization. Philos Trans R Soc Lond Series B Biol Sci 360:781-795. CrossRef

Petrides M, Pandya DN (1999) Dorsolateral prefrontal cortex: comparative cytoarchitectonic analysis in the human and the macaque brain and corticocortical connection patterns. Eur J Neurosci 11:1011-1036. CrossRef Medline

Polanía R, Paulus W, Antal A, Nitsche MA (2011) Introducing graph theory to track for neuroplastic alterations in the resting human brain: a transcranial direct current stimulation study. Neuroimage 54:2287-2296. CrossRef Medline

Reis J, Schambra HM, Cohen LG, Buch ER, Fritsch B, Zarahn E, Celnik PA, Krakauer JW (2009) Noninvasive cortical stimulation enhances motor skill acquisition over multiple days through an effect on consolidation. Proc Natl Acad Sci U S A 106:1590-1595. CrossRef Medline

Short EB, Borckardt J, Anderson BS, Frohman H, Beam W, Reeves ST, George MS (2011) Ten sessions of adjunctive left prefrontal rTMS significantly reduces fibromyalgia pain: a randomized, controlled pilot study. Pain 152:2477-2484. CrossRef Medline

Smith SM (2002) Fast, robust automated brain extraction. Hum Brain Mapp 17:143-155. CrossRef Medline

Smith SM, Jenkinson M, Woolrich MW, Beckmann CF, Behrens TE, Johansen-Berg H, Bannister PR, De Luca M, Drobnjak I, Flitney DE, Niazy RK, Saunders J, Vickers J, Zhang Y, De Stefano N, Brady JM, Matthews PM (2004) Advances in functional and structural MR image anal- ysis and implementation as FSL. Neuroimage 23:S208-S219. CrossRef Medline

Stagg CJ, Nitsche MA (2011) Physiological basis of transcranial direct current stimulation. Neuroscientist 17:37-53. CrossRef Medline

Stagg CJ, O’Shea J, Kincses ZT, Woolrich M, Matthews PM, Johansen-Berg H (2009) Modulation of movement-associated cortical activation by transcranial direct current stimulation. Eur J Neurosci 30:1412-1423. CrossRef Medline

Stagg CJ, Jayaram G, Pastor D, Kincses ZT, Matthews PM, Johansen-Berg H (2011) Polarity and timing-dependent effects of transcranial direct current stimulation in explicit motor learning. Neuropsychologia 49:800-804. CrossRef Medline

Wachter D, Wrede A, Schulz-Schaeffer W, Taghizadeh-Waghefi A, Nitsche MA, Kutschenko A, Rohde V, Liebetanz D (2011) Transcranial direct current stimulation induces polarity-specific changes of cortical blood perfusion in the rat. Exp Neurol 227:322-327. CrossRef Medline

Woolrich MW, Ripley BD, Brady M, Smith SM (2001) Temporal autocorrelation in univariate linear modelling of FMRI data. Neuroimage 14: 1370-1386. CrossRef Medline

Woolrich MW, Jbabdi S, Patenaude B, Chappell M, Makni S, Behrens T, Beckmann C, Jenkinson M, Smith SM (2009) Bayesian analysis of neuroimaging data in FSL. Neuroimage 45:S173-S186. CrossRef Medline

Zheng X, Alsop DC, Schlaug G (2011) Effects of transcranial direct current stimulation (tDCS) on human regional cerebral blood flow. Neuroimage 58:26-33. CrossRef Medline 